hep-th/9808046

IPM-98

\title{
Interaction of Branes at Angles in M(atrix) Model
}

\author{
Kamran Kaviani ${ }_{a, b}$, Shahrokh Parvizi ${ }_{a}$, Amir H. Fatollahi ${ }_{a, c}$ \\ a)Institute for Studies in Theoretical Physics and Mathematics (IPM), \\ P.O.Box 19395-5531, Tehran, Iran \\ b)Department of Physics, Az-zahra University, \\ P.O.Box 19834, Tehran, Iran \\ c)Department of Physics, Sharif University of Technology, \\ P.O.Box 11365-9161, Tehran, Iran \\ E-mails: kaviani, parvizi, fath@theory.ipm.ac.ir
}

\begin{abstract}
Interactions of relatively rotated Dp-branes in 1, 2, 3 and 4 angles in $\mathrm{M}$ (atrix) model are calculated and it is found to be in agreement with string theory calculations. In 4 angles case the agreement is achieved after subtracting the contribution of the single chiral fermionic zero mode.
\end{abstract}

M(atrix) model as a non-perturbative formulation of M-theory theory has activated a great effort in studies for finding the unified string theory. The model is the dimensional reduction of the $9+1$ dimensional $\mathcal{N}=1, U(N)$ SYM to $0+1$ dimension. This theory has passed several checks [2].

The aim of this letter is the calculation of interactions of rotated branes by the model and comparing them with the known results to provide a further test for this model. In summary we have found that the result of $\mathrm{M}$ (atrix) model calculation is in agreement with similar ones in string theory. In the more novel case of rotated 4-branes with four angles it is found that by removing the chiral fermionic zero mode in defining the determinant one can recover the contribution $R(-1)^{F}$ of string theory to get the correct result.

Rotated D-branes are important due to their role in constructing configurations with different fraction of preserved supersymmetry [3, 4]. Also in the 4 angles case they have been considered in the context of anomalous creation of fundamental strings [0, 6, 0].

The BFSS Lagrangian in units $2 \pi \alpha^{\prime}=1$ is [1]

$$
\begin{array}{r}
L=\frac{1}{2 g_{s}} \operatorname{Tr}\left(D_{t} X_{i} D_{t} X_{i}+i \theta^{T} D_{t} \theta+\frac{1}{2}\left[X_{i}, X_{j}\right]^{2}+\theta^{T} \gamma_{i}\left[\theta, X_{i}\right]\right), \\
D_{t} *=\partial_{t} *-i\left[A_{0}, *\right], \quad i, j=1, \ldots, 9,
\end{array}
$$


where $X_{i}$ and $\theta$ are bosonic and fermionic $S O(9)$ hermitian $n \times n$ matrices. The static classical equations of motion with $A_{0}=\theta=0$ are

$$
\sum_{i}\left[X^{i},\left[X^{i}, X^{j}\right]\right]=0
$$

Every configuration with $\left[X^{i}, X^{j}\right] \sim 1$ and with the other $X^{\prime}$ 's vanishing are solutions of (2).

Solutions which can be interpreted as $\mathrm{D} p$-brane have the form

$$
X_{i}^{c l}=\left(B_{1}, B_{2}, \ldots, B_{p}, 0, \ldots, 0\right), \quad A_{0}^{c l}=\theta=0
$$

where $B_{1}, \ldots, B_{p}$ are $n \times n$ matrices with $n$ large, with the commutation relations

$$
\left[B_{a}, B_{b}\right]=i c_{a b} \mathbf{1}, \quad a, b=1,2, \ldots, p
$$

By a proper rotation the anti-symmetric matrix $c_{a b}$ can be brought to the Jordan form

$$
c_{a b}=\left(\begin{array}{ccccc}
0 & \omega_{1} & & & \\
-\omega_{1} & 0 & & & \\
& & \ddots & & \\
& & & 0 & \omega_{l} \\
& & & -\omega_{l} & 0
\end{array}\right),
$$

where $2 l \equiv p=2,4,6,8$.

These solutions can be represented by

$$
\left\{\begin{array}{l}
X_{2 i-1}=1_{n_{1}} \otimes 1_{n_{2}} \otimes \ldots \otimes \frac{L_{2 i-1}}{\sqrt{2 \pi n_{i}}} q_{i} \otimes 1_{n_{i+1}} \otimes \ldots \otimes 1_{n_{l}}, \\
X_{2 i}=1_{n_{1}} \otimes 1_{n_{2}} \otimes \ldots \otimes \frac{L_{2 i}}{\sqrt{2 \pi n_{i}}} p_{i} \otimes 1_{n_{i+1}} \otimes \ldots \otimes 1_{n_{l}}, \quad l \geq i \\
X^{i}=0, \quad i>2 l=p,
\end{array}\right.
$$

where $n_{1} n_{2} \ldots n_{l}=n$ and $L_{a}$ 's are compactification radii, with commutation relations

$$
\left[q_{i}, p_{j}\right]=i \delta_{i j} 1_{n_{i}}
$$

The eigenvalues of $q, p$ are uniformly distributed as

$$
-\sqrt{\frac{\pi n_{i}}{2}} \leq q_{i}, p_{i} \leq \sqrt{\frac{\pi n_{i}}{2}}
$$

So the extension of solutions along $X_{i}$ axis is $L_{i} \rightarrow \infty$. Thus one can obtain

$$
\left[X_{2 i-1}, X_{2 i}\right]=\frac{i}{2 \pi n_{i}} L_{2 i-1} L_{2 i} 1_{n}, \quad 0 \leq i \leq l
$$

and correspondingly $\frac{n^{\frac{1}{l}}}{L_{2 i-1} L_{2 i}}=\frac{1}{2 \pi \omega_{i}}$ by $n_{i} \sim n^{\frac{1}{l}}$ [8]. 
The configurations with four rotation angles can be obtained from the block-diagonal matrices with two identical blocks describing a pair of $\mathrm{D} p$-branes. Translating along the $(p+5)$-th axis by the distance $r$ from each other and rotating in opposite directions through the angles $\psi_{4} / 2, \psi_{3} / 2, \psi_{2} / 2$ and $\psi_{1} / 2$, we obtain the configuration of two rotated $\mathrm{D} p$-branes

$$
\begin{aligned}
& X_{a}^{c l}=\left(\begin{array}{cc}
B_{a} & 0 \\
0 & B_{a}
\end{array}\right), \quad a=1, \ldots, p-4, \\
& X_{p-3}^{c l}=\left(\begin{array}{cc}
B_{p-3} \cos \frac{\psi_{4}}{2} & 0 \\
0 & B_{p-3} \cos \frac{\psi_{4}}{2}
\end{array}\right), \quad X_{p-2}^{c l}=\left(\begin{array}{cc}
B_{p-2} \cos \frac{\psi_{3}}{2} & 0 \\
0 & B_{p-2} \cos \frac{\psi_{3}}{2}
\end{array}\right) \text {, } \\
& X_{p-1}^{c l}=\left(\begin{array}{cc}
B_{p-1} \cos \frac{\psi_{2}}{2} & 0 \\
0 & B_{p-1} \cos \frac{\psi_{2}}{2}
\end{array}\right), \quad X_{p}^{c l}=\left(\begin{array}{cc}
B_{p} \cos \frac{\psi_{1}}{2} & 0 \\
0 & B_{p} \cos \frac{\psi_{1}}{2}
\end{array}\right) \text {, } \\
& X_{p+1}^{c l}=\left(\begin{array}{cc}
B_{p} \sin \frac{\psi_{1}}{2} & 0 \\
0 & -B_{p} \sin \frac{\psi_{1}}{2}
\end{array}\right), \quad X_{p+2}^{c l}=\left(\begin{array}{cc}
B_{p-1} \sin \frac{\psi_{2}}{2} & 0 \\
0 & -B_{p-1} \sin \frac{\psi_{2}}{2}
\end{array}\right) \text {, } \\
& X_{p+3}^{c l}=\left(\begin{array}{cc}
B_{p-2} \sin \frac{\psi_{3}}{2} & 0 \\
0 & -B_{p-2} \sin \frac{\psi_{3}}{2}
\end{array}\right), \quad X_{p+4}^{c l}=\left(\begin{array}{cc}
B_{p-3} \sin \frac{\psi_{4}}{2} & 0 \\
0 & -B_{p-3} \sin \frac{\psi_{4}}{2}
\end{array}\right) \text {, } \\
& X_{p+5}^{c l}=\left(\begin{array}{cc}
\frac{r}{2} & 0 \\
0 & -\frac{r}{2}
\end{array}\right), \quad A_{0}^{c l}=X_{i}^{c l}=0, \quad i=p+6, \ldots, 9 .
\end{aligned}
$$

To calculate the one-loop effective action it is convenient to work with a compact form of (1) after the Wick rotation $t \rightarrow i t$ and $A_{0} \rightarrow-i A_{0}$

$$
\begin{array}{r}
L=\frac{1}{g_{s}} \operatorname{Tr}\left(\frac{1}{4}\left[X_{\mu}, X_{\nu}\right]^{2}+\frac{1}{2} \theta^{T} \gamma^{\mu}\left[X_{\mu}, \theta\right]\right), \\
\mu, \nu=0,1, \ldots, 9, \quad X_{0}=i \partial_{t}+A_{0},
\end{array}
$$

where sums are with Euclidean metric and $\gamma_{0}=-i$.

The one-loop effective action $W$ with the backgrounds $\theta=A_{0}^{c l}=0$ was calculated in [9, 10]. In our notation the bosonic, fermionic and ghost contribution can be written as,

$$
W=-\ln \left(\operatorname{det}^{-\frac{1}{2}}\left(P_{\lambda}^{2} \delta_{\mu \nu}-2 i F_{\mu \nu}\right) \cdot \operatorname{det}^{\frac{1}{2}}\left(\partial_{t}+\sum_{i=1}^{9} P_{i} \gamma_{i}\right) \cdot \operatorname{det}\left(P_{\lambda}^{2}\right)\right),
$$

with $P_{\nu} *=\left[X_{\mu}^{c l}, *\right], F_{\mu \nu} *=\left[f_{\mu \nu}, *\right], f_{\mu \nu}=i\left[X_{\mu}^{c l}, X_{\nu}^{c l}\right]$,

$$
P_{\lambda}^{2}=-\partial_{t}^{2}+\sum_{i=1}^{9} P_{i}^{2} \text { and } \quad F_{0 i}=0
$$

which the last equality is true for static configurations with $A_{0}^{c l}=0$. 
It is worth mentioning that the configurations with $F_{\mu \nu} \equiv 0$ for all $\mu, \nu$ have vanishing quantum corrections due to the algebra

$$
W \sim \operatorname{Tr} \log \left(P_{\lambda}^{2}\right)\left(\frac{10}{2}-\frac{16}{4}-1\right)=0 .
$$

By setting $\omega_{1}=\omega_{2}=\ldots=\omega$ one finds

$$
\begin{aligned}
f_{2 a-1,2 a}= & -\omega \otimes 1, \quad a=1, \ldots, l-2, \\
f_{p-3, p-2}= & -\omega \cos \frac{\psi_{3}}{2} \cos \frac{\psi_{4}}{2} \otimes 1, \quad f_{p-3, p+3}=-\omega \cos \frac{\psi_{4}}{2} \sin \frac{\psi_{3}}{2} \otimes \sigma_{3}, \\
f_{p-2, p+4}= & \omega \cos \frac{\psi_{3}}{2} \sin \frac{\psi_{4}}{2} \otimes \sigma_{3}, \quad f_{p+3, p+4}=\omega \sin \frac{\psi_{3}}{2} \sin \frac{\psi_{4}}{2} \otimes 1, \\
f_{p-1, p}= & -\omega \cos \frac{\psi_{2}}{2} \cos \frac{\psi_{1}}{2} \otimes 1, \quad f_{p-1, p+1}=-\omega \cos \frac{\psi_{2}}{2} \sin \frac{\psi_{1}}{2} \otimes \sigma_{3}, \\
f_{p, p+2}= & \omega \cos \frac{\psi_{1}}{2} \sin \frac{\psi_{2}}{2} \otimes \sigma_{3}, \quad f_{p+1, p+2}=\omega \sin \frac{\psi_{1}}{2} \sin \frac{\psi_{2}}{2} \otimes 1, \\
& \text { otherwise } \quad f_{a b}=0,
\end{aligned}
$$

where $\sigma_{3}$ is the Pauli matrix. Then

$$
\begin{aligned}
{\left[P_{p-3}, P_{p+3}\right]=} & i \omega \cos \frac{\psi_{4}}{2} \sin \frac{\psi_{3}}{2} \otimes \Sigma_{3}, \quad\left[P_{p-2}, P_{p+4}\right]=-i \omega \cos \frac{\psi_{3}}{2} \sin \frac{\psi_{4}}{2} \otimes \Sigma_{3}, \\
{\left[P_{p-1}, P_{p+1}\right]=} & i \omega \cos \frac{\psi_{2}}{2} \sin \frac{\psi_{1}}{2} \otimes \Sigma_{3}, \quad\left[P_{p}, P_{p+2}\right]=-i \omega \cos \frac{\psi_{1}}{2} \sin \frac{\psi_{2}}{2} \otimes \Sigma_{3}, \\
& \text { otherwise } \quad F_{a b}=0, \quad P_{p+5}=\frac{r}{2} \otimes \Sigma_{3},
\end{aligned}
$$

where $\Sigma_{3} *=\left[1 \otimes \sigma_{3}, *\right] . \Sigma_{3}$ has $2,-2,0$ and 0 as eigenvalues.

We define the following parameters for later conventions:

$$
\begin{array}{ll}
U_{1}=2 \omega \cos \frac{\psi_{4}}{2} \sin \frac{\psi_{3}}{2}, & U_{2}=2 \omega \cos \frac{\psi_{3}}{2} \sin \frac{\psi_{4}}{2} \\
U_{3}=2 \omega \cos \frac{\psi_{2}}{2} \sin \frac{\psi_{1}}{2}, & U_{4}=2 \omega \cos \frac{\psi_{1}}{2} \sin \frac{\psi_{2}}{2} .
\end{array}
$$

So one finds for (10)

$$
\begin{aligned}
W=-\ln ( & \operatorname{det}^{-1}\left(P_{\lambda}^{2}\right) \\
& \times \operatorname{det}^{-\frac{1}{2}}\left(P_{\lambda}^{2}-2 U_{1}\right) \operatorname{det}^{-\frac{1}{2}}\left(P_{\lambda}^{2}+2 U_{1}\right) \\
& \times \operatorname{det}^{-\frac{1}{2}}\left(P_{\lambda}^{2}-2 U_{2}\right) \operatorname{det}^{-\frac{1}{2}}\left(P_{\lambda}^{2}+2 U_{2}\right) \\
& \times \operatorname{det}^{-\frac{1}{2}}\left(P_{\lambda}^{2}-2 U_{3}\right) \operatorname{det}^{-\frac{1}{2}}\left(P_{\lambda}^{2}+2 U_{3}\right) \\
& \times \operatorname{det}^{-\frac{1}{2}}\left(P_{\lambda}^{2}-2 U_{4}\right) \operatorname{det}^{-\frac{1}{2}}\left(P_{\lambda}^{2}+2 U_{4}\right) \\
& \left.\times \operatorname{det}\left(P_{\lambda}^{2}\right) \operatorname{det}^{\frac{1}{2}}\left(\partial_{t}+\sum_{i=1}^{9} P_{i} \gamma_{i}\right)\right) .
\end{aligned}
$$


In the cases of our interest we have $\left[X_{\mu}^{c l}, f_{\mu \nu}\right]=c-$ number, and so $P_{\lambda}^{2}$ and $F_{\mu \nu}$ are simultaneously diagonalisable.

\section{1) Four angles}

The four angles case is allowed only for 4-branes, $p=4=2 l$. Also in the four angles case the second term in (10) can not bring to the Klein-Gordon form because of the absence tenth $16 \times 16$ matrix which anti-commutes with all the $\gamma_{i}$ 's. But an expansion for small $r$ is possible and one finds

$$
\begin{aligned}
& -\ln \operatorname{det}^{\frac{1}{2}}\left(\partial_{t}+\sum_{i=1}^{9} P_{i} \gamma_{i}\right)=-\frac{1}{2} \operatorname{Tr} \ln \left(\partial_{t}+\sum_{i=1}^{8} P_{i} \gamma_{i}+P_{9} \gamma_{9}\right)= \\
& -\frac{1}{4} \operatorname{Tr} \ln \left(P_{\lambda}^{2}+\frac{i}{2} F_{i j} \gamma_{i j}\right)-\frac{r}{2} \operatorname{Tr}\left(\frac{1}{\partial_{t} \gamma_{9}+\sum_{i=1}^{8} P_{i} \gamma_{9} \gamma_{i}}\right)+O\left(r^{2}\right),
\end{aligned}
$$

which the last trace is shown to vanish in the Appendix.

The eigenvalues of $P_{\lambda}^{2}$ can be read from (14). Zero eigenvalues of $\Sigma_{3}$ will not have any contribution to the effective action because of (12). The other two eigenvalues force $\left(P_{p-3}, P_{p+3}\right),\left(P_{p-2}, P_{p+4}\right),\left(P_{p-1}, P_{p+1}\right)$ and $\left(P_{p}, P_{p+2}\right)$ to behave as harmonic oscillators with their related frequencies to be read from (14). The eigenvalues of $P_{\lambda}^{2}$ are

$$
E=r^{2}+q_{0}^{2}+2 \sum_{a=1}^{4} U_{a}\left(k_{a}+\frac{1}{2}\right),
$$

with $q_{0}$ as eigenvalues of $i \partial_{t}$, and $k_{a}$ as harmonic oscillator numbers.

Using Schwinger's time representation, one finds the one-loop effective action $W$ as,

$$
\begin{aligned}
& W=\int d t \int_{0}^{\infty} \frac{d s}{s} \mathrm{e}^{-s\left(q_{0}^{2}+r^{2}\right)} \sum_{k_{1}, \cdots, k_{4}=0}^{\infty} \mathrm{e}^{-s \sum_{a=1}^{4} U_{a}\left(2 k_{a}+1\right)} \\
& \times\left[2 \sum_{a=1}^{4}\left(\mathrm{e}^{-2 s U_{a}}+\mathrm{e}^{2 s U_{a}}\right)-\sum_{\mathbf{s}_{1}, \ldots, \mathbf{s}_{4}= \pm 1} \mathrm{e}^{-s \sum_{a=1}^{4} U_{a} \mathbf{s}_{a}}\right],
\end{aligned}
$$

where $\mathbf{s}_{1,2,3,4}$ are the eigenvalues of the commutative matrices $i \gamma_{17}, i \gamma_{82}, i \gamma_{35}$, i $\gamma_{64}$ respectively and the terms in the last bracket come from bosons and fermions respectively. In the above expression for $W$ one can specify the contribution of a chiral fermionic zero mode defined by

$$
\left(\sum_{i=1}^{8} P_{i} \gamma_{i}\right) \theta_{0}=0
$$

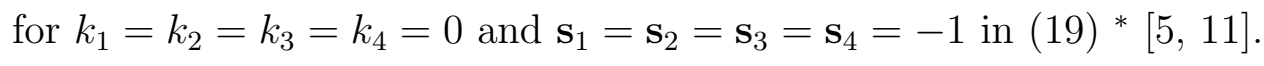

*By chirality we mean in the 8 dimensional subspace defined by the two 4-branes as

$$
\left(i \gamma_{17}\right)\left(i \gamma_{82}\right)\left(i \gamma_{35}\right)\left(i \gamma_{64}\right) \theta_{0}=(-1)^{4} \theta_{0}=\gamma_{1} \gamma_{2} \gamma_{3} \gamma_{4} \gamma_{5} \gamma_{6} \gamma_{7} \gamma_{8} \theta_{0}=\gamma_{9} \theta_{0}
$$


To calculate the one-loop effective action one should project out the contribution of the chiral fermionic zero mode [12, 13] to be done here as i]

$$
W^{\prime}=W-\int d t \int_{0}^{\infty} \frac{d s}{s} \mathrm{e}^{-s\left(q_{0}^{2}+r^{2}\right)}(-1) .
$$

By putting $\omega<<1$ one finds,

$$
\begin{aligned}
W^{\prime}= & \int d t \int_{0}^{\infty} \frac{d s}{s}\left(\frac{\pi}{s}\right)^{\frac{1}{2}} \mathrm{e}^{-s r^{2}} \\
& \times \frac{\sum_{a=1}^{4} \cos \left(2 \psi_{a}\right)-4 \prod_{a=1}^{4} \cos \psi_{a}+4 \prod_{a=1}^{4} \sin \psi_{a}+\mathcal{O}\left(s^{2}\right)}{4 \prod_{a=1}^{4} \sin \psi_{a}},
\end{aligned}
$$

and finally

$$
W^{\prime}=-2 \sqrt{\pi} \int d t \frac{\sum_{a=1}^{4} \cos \left(2 \psi_{a}\right)-4 \prod_{a=1}^{4} \cos \psi_{a}+4 \prod_{a=1}^{4} \sin \psi_{a}}{4 \prod_{a=1}^{4} \sin \psi_{a}}|r|,
$$

in agreement with string theory calculations [3].

The above interaction vanishes in $\psi_{1}+\psi_{2}+\psi_{3}+\psi_{4}=0(\bmod 2 \pi)$ cases, signalling enhancement of SUSY. An equivalent result is obtained in [3] by considering interactions of rotated D $p$-branes, and in [4] by studying the SUSY algebra for rotated M-objects.

\section{2) Three angles}

By putting $\psi_{4}=0$ in (8) one finds the relevant configuration and commutation relations for the 3 angles case. Again the 3 angles are accessible only for $p=4$.

The eigenvalues of $P_{\lambda}^{2}$ can be read from (14). Zero eigenvalues of $\Sigma_{3}$ will not have any contribution to the effective action because of (12). As the four angles case one has harmonic oscillators with their related frequencies to be read from (14). The eigenvalues of $P_{\lambda}^{2}$ are

$$
E_{\mathbf{q}, \mathbf{p}, \mathbf{k}}=r^{2}+q_{0}^{2}+p_{1}^{2} \cos ^{2} \frac{\psi_{3}}{2}+2 \sum_{a=1,3,4} U_{a}\left(k_{a}+\frac{1}{2}\right)
$$

with $q_{0}$ and $p_{1}$ as eigenvalues of $i \partial_{t}$ and $P_{2}$ respectively, and $k_{a}$ as harmonic oscillator numbers.

The one-loop effective action is given by (16) and using $\mathrm{f}$

$$
-\ln \operatorname{det}^{\frac{1}{2}}\left(\partial_{t}+\sum_{i=1}^{9} P_{i} \gamma_{i}\right)=-\frac{1}{4} \operatorname{Tr} \ln \left(P_{\lambda}^{2}+\frac{i}{2} F_{i j} \gamma_{i j}\right)
$$

one can calculate $W$

$$
W \sim \int d t \frac{\sum_{a=1}^{3} \cos \left(2 \psi_{a}\right)-4 \prod_{a=1}^{3} \cos \psi_{a}}{\cos \frac{\psi_{3}}{2} \prod_{a=1}^{3} \sin \psi_{a}} \ln |r|
$$

\footnotetext{
${ }^{\dagger}$ The same is true for D0-D8-brane system studied in [14].

${ }^{\ddagger}$ Here equality is exact in contrast to $(17)$ because at least one of $P_{i}$ 's is zero.
} 
which is in agreement with string theory results [3].

\section{3) Two angles}

By putting $\psi_{4}=\psi_{3}=0$ in (8) the eigenvalues of $P_{\lambda}^{2}$ are $(2 l=p)$

$$
E_{\mathbf{q}, \mathbf{p}, \mathbf{k}}=r^{2}+q_{0}^{2}+\sum_{i=1}^{l-1}\left(q_{i}^{2}+p_{i}^{2}\right)+2 \sum_{a=3,4} U_{a}\left(k_{a}+\frac{1}{2}\right) .
$$

where $q$ and $p$ 's are the eigenvalues of those $P$ 's which do not behave as harmonic oscillators. After calculations similar to the above cases, for large separation between branes we find:

$$
W \sim \frac{\left(\cos \psi_{1}-\cos \psi_{2}\right)^{2}}{\sin \psi_{2} \sin \psi_{1}}\left(\frac{1}{r^{5-p}}\right)+\cdots,
$$

which is in agreement with string theory results [3].

The above interaction vanishes in $\psi_{2}=\psi_{1}$ cases, signalling enhancement of SUSY [3, 四.

\section{4) One angle}

By putting $\psi_{4}=\psi_{3}=\psi_{2}=0$ one finds the eigenvalues of $P_{\lambda}^{2}$ as

$$
E_{\mathbf{q}, \mathbf{p}, k}=r^{2}+q_{0}^{2}+\sum_{i=1}^{l-1}\left(q_{i}^{2}+p_{i}^{2}\right)+\cos ^{2} \frac{\psi_{1}}{2} q_{l}^{2}+2 U_{3}\left(k_{3}+\frac{1}{2}\right) .
$$

For large separation between branes we find:

$$
W \sim \tan \frac{\psi_{1}}{2} \sin ^{2} \frac{\psi_{1}}{2} \frac{1}{r^{6-p}}+\cdots,
$$

again in agreement with string theory results [3, 15].

\section{Acknowledgement}

It is a pleasure to thank M.M. Sheikh-Jabbari for useful discussions.

\section{Appendix}

Here we calculate the trace appeared in (17).

$$
\operatorname{Tr}\left(\frac{1}{\partial_{t} \gamma_{9}+\sum_{i=1}^{8} P_{i} \gamma_{9} \gamma_{i}}\right)=\operatorname{Tr}\left(\frac{\gamma_{9}}{\partial_{t}+\sum_{i=1}^{8} P_{i} \gamma_{i}}\right) \text {. }
$$

Multiplying the denominator and the nominator by $-\partial_{t}+\sum_{i=1}^{8} P_{i} \gamma_{i}$ and using Schwinger's time representation one finds

$\operatorname{Tr}\left(\frac{\gamma_{9}}{\partial_{t}+\sum_{i=1}^{8} P_{i} \gamma_{i}}\right)=\operatorname{Tr}\left(\gamma_{9}\left(-\partial_{t}+\sum_{i=1}^{8} P_{i} \gamma_{i}\right) \times \int_{0}^{\infty} d s \exp \left\{-s\left[-\partial_{t}^{2}+P_{i}^{2}+\frac{i}{2} \sum_{i, j=1}^{8} F_{i j} \gamma_{i j}\right]\right\}\right)$.

The term proportional to $-\partial_{t}$ vanishes because it is an odd function with respect to the eigenvalues of $\partial_{t}$ in the trace. The next 8 terms containing $\gamma_{i}$ 's give zero because they are multiplications of odd numbers of $\gamma$ matrices in the even number of $\gamma$ of exponential, by knowing $\gamma_{9}=\gamma_{1} \gamma_{2} \gamma_{3} \gamma_{4} \gamma_{5} \gamma_{6} \gamma_{7} \gamma_{8}$. 


\section{References}

[1] T. Banks, W. Fischler, S. H. Shenker and L. Susskind, Phys. Rev. D55, 5112 (1997), hep-th/9610043.

[2] T. Banks, "Matrix Theory" and references therein, hep-th/9710231.

[3] M.M. Sheikh-Jabbari, Phys. Lett. B420 (1998) 279, hep-th/9710121,

[4] N. Ohta and P.K. Townsend, "Supersymmetry of M-branes at Angles", hep-th/9710129.

[5] P.M. Ho and Y.S. Wu, "Brane Creation in M(atrix) Theory", hep-th/9708137.

[6] N. Ohta, T. Shimizu and J.-G. Zhou, "Creation of Fundamental String in M(atrix) Theory", hep-th/9710218.

[7] T. Kitao, N. Ohta and J.-G. Zhou, "Fermionic Zero Mode and String Creation between D4-Branes at Angles", hep-th/9801135.

[8] T. Banks, N. Seiberg and S. Shenker, Nucl. Phys. B490, 91, (1997), hep-th/9612157.

[9] E.S. Fradkin and A.A. Tseytlin, Nucl. Phys. B227, 252, (1983).

[10] I. Chepelev and A.A. Tseytlin, Phys. Rev. D56, 3672, (1997), hep-th/9704127.

[11] P.M. Ho, M. Li and Y.S. Wu, "p-p'Strings in M(atrix) Theory", hep-th/9706073.

[12] M. Billo, P. Di Vecchia, M. Frau, A. Lerda, R. Russo and S. Sciuto, "The Lorentz Force between D0 and D6 Branes in String Theory and M(atrix) Theory", hepth/9805091.

[13] J.W. Negele and H. Orland, "Quantum Many-Particle Systems", Addison-Wesley Publ. Comp. (1988).

[14] J.M. Pierre, Phys. Rev. D57 (1998) 1250, hep-th/9705110.

[15] H. Arfaei and M.M. Sheikh-Jabbari, Phys. Lett. B394, 288, (1997), hep-th/9608167. 